\section{SYNTHESES OF DERIVATIVES OF 4'-DEOXYMYCAMINOSYL TYLONOLIDE AND MYCAMINOSYL TYLONOLIDE MODIFIED AT C-23}

Sir:

In the foregoing communication ${ }^{1)}$ we reported the synthesis of 4'-deoxymycaminosyl tylonolide (1a) which showed fairly strong antibacterial activity. This paper describes the syntheses of derivatives of $\mathbf{1 a}$ and of mycaminosyl tylonolide (1b) modified at C-23.

Treatment of the diethylacetal (2a) of 1a with acetic anhydride in acetonitrile as described previously $^{1)}$ gave the $2^{\prime}$-O-acetyl derivative (3) $\left(93 \%\right.$ ), m.p. $187 \sim 190^{\circ} \mathrm{C},[\alpha]_{\mathrm{D}}^{23}+28^{\circ}$ (c 1, chloroform). Acylation of $\mathbf{3}$ with acetyl chloride, propanoyl chloride, $n$ - or isobutanoyl chloride and benzoyl chloride in pyridine $\left(-20^{\circ} \mathrm{C}, \simeq 1\right.$ hour $)$ followed by treatment of the acyl derivatives with methanol $\left(50^{\circ} \mathrm{C}\right.$, overnight $\left.{ }^{1)}\right)$ to remove the $2^{\prime}$ $\mathrm{O}$-acetyl group, gave 23-O-acetyl (4), 23-Opropanoyl (5), 23-O-n-butanoyl (6), 23-O-isobutanoyl (7) and 23-O-benzoyl (8) derivatives in higher than $90 \%$ yields (after purification) in all cases; the values of $[\alpha]_{\mathrm{D}}^{19}(c 1$, chloroform) were: 4, $+10^{\circ} ; \mathbf{5},+13^{\circ} ; \mathbf{6},+13^{\circ} ; \mathbf{7},+17^{\circ} ; \mathbf{8},+11^{\circ}$. The acylated positions (the 23-hydroxyl group) were confirmed, in their ${ }^{1} \mathrm{H}$ NMR spectra $(4 \sim 8)$, by the appearance of two proton signals assignable to 23- $\mathrm{CH}_{2} \mathrm{O}$-acyl at $\delta 4.2 \sim 4.4$ (cf. the shift values of $\mathrm{H}-3$ and -23 of 3,23-di-O-acetylmycaminosyl tylonolide diethylaceta $\left.{ }^{2)}\right)$. Deacetalation of the acyl derivatives with $0.1 \mathrm{M}$ aqueous hydrochloric acid in acetonitrile $\left(3: 2 \mathrm{v} / \mathrm{v}, 25^{\circ} \mathrm{C}, 1\right.$ hour $)$ gave the desired 23-O-acetyl- (9a, m.p. 106 $108^{\circ} \mathrm{C},[\alpha]_{\mathrm{D}}^{1 \theta}-12^{\circ}(c, 1$, chloroform $\left.)\right), 23-O$-propanoyl- $\left(10 \mathrm{a},-6^{\circ}\right), 23-O-n$-butanoyl- $\left(11 \mathrm{a},-9^{\circ}\right)$, 23-O-isobutanoyl- $\left(\mathbf{1 2 a},-6^{\circ}\right)$ and 23-O-benzoyl$4^{\prime}$-deoxymycaminosyl tylonolides $\left(\mathbf{1 3 a},-19^{\circ}\right)$ in higher than $90 \%$ yields (after purification). As the reference compounds, 23-O-acetyl- (9b), 23-O-propanoyl- (10b), 23-O-n-butanoyl- (11b), 23-O-isobutanoyl- (12b) and 23-O-benzoylmycaminosyl tylonolides (13b) were also prepared similarly from mycaminosyl tylonolide diethylacetal $^{2)}$ (2b) by way of $2^{\prime}, 4^{\prime}-O$-acetylation, selective 23-O-acylation, $\operatorname{de}\left(2^{\prime}, 4^{\prime}\right.$-di- $O$-acetyl $)$ ation and deacetalation.

C-23-halo derivatives of 1 a were next prepared. Treatment of $\mathbf{2 a}$ with triphenylphosphine and carbon tetrachloride (molar ratio of $1: 2.2: 1.1$ )

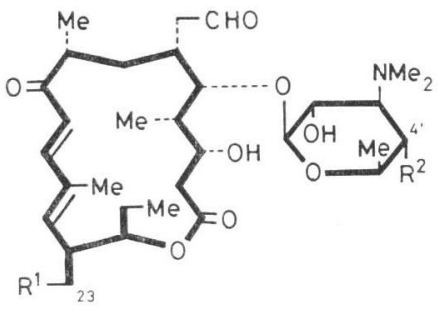

\begin{tabular}{|c|c|c|c|c|c|}
\hline & $\mathrm{R}^{1}$ & $\mathrm{R}^{2}$ & & $\mathrm{R}^{1}$ & $\mathrm{R}^{2}$ \\
\hline $1 \mathrm{a}$ & $\mathrm{OH}$ & $\mathrm{H}$ & $13 b$ & $\mathrm{OCOC}_{6} \mathrm{H}_{5}$ & $\mathrm{OH}$ \\
\hline 1b & $\mathrm{OH}$ & $\mathrm{OH}$ & $17 \mathbf{a}$ & $\mathrm{Cl}$ & $\mathrm{H}$ \\
\hline $9 \mathrm{a}$ & OAc & $\mathrm{H}$ & $17 \mathrm{~b}$ & $\mathrm{Cl}$ & $\mathrm{OH}$ \\
\hline $9 b$ & OAc & $\mathrm{OH}$ & $18 \mathrm{a}$ & $\mathrm{Br}$ & $\mathrm{H}$ \\
\hline $10 \mathrm{a}$ & $\mathrm{OCOCH}_{2} \mathrm{CH}_{3}$ & $\mathrm{H}$ & $18 b$ & $\mathrm{Br}$ & $\mathrm{OH}$ \\
\hline $10 \mathrm{~b}$ & $\mathrm{OCOCH}_{2} \mathrm{CH}_{3}$ & $\mathrm{OH}$ & 19a & I & $\mathrm{H}$ \\
\hline $11 \mathrm{a}$ & $\mathrm{OCO}\left(\mathrm{CH}_{2}\right)_{2} \mathrm{CH}_{3}$ & $\mathrm{H}$ & $19 \mathrm{~b}$ & I & $\mathrm{OH}$ \\
\hline 11b & $\mathrm{OCO}\left(\mathrm{CH}_{2}\right)_{2} \mathrm{CH}_{3}$ & $\mathrm{OH}$ & $21 \mathrm{a}$ & $\mathrm{O} \sim \mathrm{THF}$ & $\mathrm{H}$ \\
\hline $12 \mathrm{a}$ & $\mathrm{OCOCH}\left(\mathrm{CH}_{3}\right)_{2}$ & $\mathrm{H}$ & $21 b$ & $\mathrm{O} \sim \mathrm{THF}$ & $\mathrm{OH}$ \\
\hline $12 b$ & $\mathrm{OCOCH}\left(\mathrm{CH}_{3}\right)_{2}$ & $\mathrm{OH}$ & $22 a$ & $\mathrm{O} \sim \mathrm{THP}$ & $\mathrm{H}$ \\
\hline $13 \mathrm{a}$ & $\mathrm{OCOC}_{6} \mathrm{H}_{5}$ & $\mathrm{H}$ & $22 b$ & $\mathrm{O} \sim \mathrm{THP}$ & $\mathrm{OH}$ \\
\hline
\end{tabular}

THF: Tetrahydrofuran

THP: Tetrahydropyran

in pyridine under nitrogen (room temperature, 20 hours) according to the method of ANISUZZAMAN and WHISTLER ${ }^{3)}$ gave 23-chloro-23,4'dideoxymycaminosyl tylonolide diethylacetal (14a) $(99 \%)$, m.p. $164 \sim 167^{\circ} \mathrm{C},[\alpha]_{\mathrm{D}}^{23}+12^{\circ}$ (c 1, chloroform); Found (Calcd. for $\mathrm{C}_{35} \mathrm{H}_{60} \mathrm{NO}_{2} \mathrm{Cl}$ ): C, 62.19 (62.34); H, 8.71 (8.97); N, 2.32 (2.08); $\mathrm{Cl}, 5.16(5.26) \%$. The structure was confirmed by the ${ }^{1} \mathrm{H}$ NMR spectrum of the 3 -O-acetyl derivative of $14 \mathrm{a}\left(\mathrm{H}-3: \delta 5.17 ; \mathrm{H}-3^{\prime} ; \delta 2.47\right.$, dt, $J$ $11.5 \times 2$ and $4 \mathrm{~Hz}$ ). Similarly, 23-bromo-23deoxy (15a) (95\%) and 23-deoxy-23-iodo (16a) $(87 \%$ ) derivatives of $\mathbf{2 a}$ were prepared by treating $\mathbf{2 a}$ with triphenylphosphine and carbon tetrabromide (room temperature, 1 hour) or triphenylphosphine and carbon tetraiodide (room temperature, 1 hour); $15 a:[\alpha]_{D}^{23}+31^{\circ}$ (c 1, chloroform), 16a: $[\alpha]_{\mathrm{D}}^{23}+78^{\circ}$ (c 1, chloroform); Found (Calcd. for $\mathrm{C}_{35} \mathrm{H}_{60} \mathrm{NO}_{9} \mathrm{I}$ ): C, 54.88 (54.90); $\mathrm{H}$, 7.75 (7.90); N, 1.77 (1.83); I, 16.28 (16.57\%). Deacetalation of the halo derivatives gave the corresponding 23-chloro-23,4'-dideoxy- $\left[17 \mathrm{a},[\alpha]_{\mathrm{D}}^{25}\right.$ $-8^{\circ}$ (c 1, chloroform)], 23-bromo-23,4'-dideoxy$\left(18 \mathbf{a},+11^{\circ}\right)$, and $23,4^{\prime}$-dideoxy-23-iodo-mycaminosyl tylonolides $\left(19 \mathrm{a},+56^{\circ}\right)$, in high yields. As the reference compounds, 23-chloro-23-deoxy(17b), 23-bromo-23-deoxy- (18b) and 23-deoxy- 
Table 1. Antibacterial spectra of

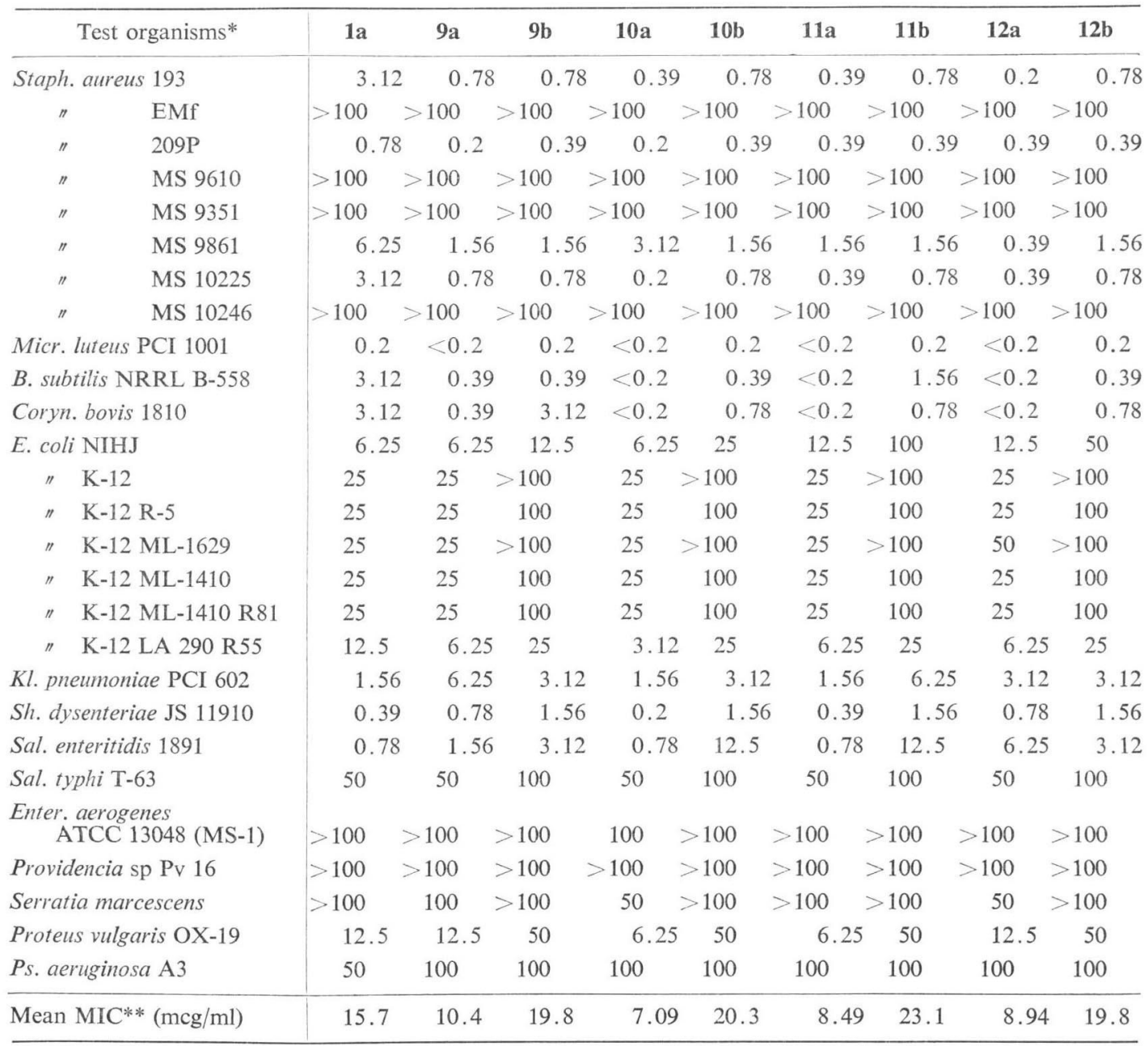

* Agar dilution streak method (nutrient agar, $37^{\circ} \mathrm{C}, 17$ hours)

** Index value being taken as 0 for $<0.2,2$ for $0.39, \ldots, 10$ for 100 , and 11 for $>10$; see. Ref. 5 . Mean

23-iodo-mycaminosyl tylonolides (19b) were prepared from the diethyl acetal of mycaminosyl tylonolide (2b) via the corresponding 23-halo-23deoxy-mycaminosyl tylonolide diethylacetals (chloro: 14b, bromo: 15b and iodo: 16b); 16b: $[\alpha]_{\mathrm{D}}^{25}+67^{\circ}$ (c 1, chloroform); Found (Calcd. for $\mathrm{C}_{35} \mathrm{H}_{60} \mathrm{NO}_{10} \mathrm{I}$ ): C, 53.52 (53.77); H, 7.49 (7.74); N, 1.90 (1.79); I, 16.38 (16.23)\%.

C-23-acetal derivatives of 1a were further prepared. Selective acetylation of $\mathbf{1 a}$ as previously described $\left(\mathrm{Ac}_{2} \mathrm{O} /\right.$ acetonitrile) gave the 2'-Oacetyl derivative (20) (94\%) having the aldehyde group free. Selective 23-O-tetrahydrofuranylation or tetrahydropyranylation of $\mathbf{2 0}$ with dihydrofuran ( 2 mole equiv. for 1a) or 3,4-dihydro-
$2 H$-pyran ( 2 mole equiv. for 1a) in dichloromethane in the presence of pyridinium $p$-toluenesulfonate $^{4)}$ (room temperature, 26 hours), followed by $\operatorname{de}\left(2^{\prime}-O\right.$-acetyl)ation by treating the derivatives with methanol $\left(50^{\circ} \mathrm{C}, 6\right.$ hours $)$ gave $23-O$ tetrahydrofuranyl- (21a) and 23-O-tetrahydropyranyl-4'-deoxymycaminosyl tylonolides (22a) in yields of 74 and $70 \%$, respectively; 21a: $[\alpha]_{D}^{12}$ $-25^{\circ}$ ( c 1, chloroform); Found (Calcd. for $\mathrm{C}_{35^{-}}$ $\mathrm{H}_{57} \mathrm{NO}_{10}$ ): C, 64.42 (64.49); $\mathrm{H}, 8.75$ (8.81); $\mathrm{N}$, $2.14(2.15) \%$. 22a: $[\alpha]_{\mathrm{D}}^{19}-17^{\circ}$ (c 1, chloroform); Found (Calcd. for $\mathrm{C}_{30} \mathrm{H}_{59} \mathrm{NO}_{10}$ ): C, 64.92 (64.94); $\mathrm{H}, 8.85(8.93) ; \mathrm{N}, 2.14(2.10 \%)$. The structures were confirmed by the ${ }^{13} \mathrm{C}$ NMR spectra.

As reference compounds, 23-O-tetrahydro- 
the products $(\mathrm{mcg} / \mathrm{ml})$.

\begin{tabular}{|c|c|c|c|c|c|c|c|c|c|c|}
\hline $13 a$ & $17 \mathrm{a}$ & $17 \mathrm{~b}$ & $18 \mathrm{a}$ & $18 \mathrm{~b}$ & 19a & $19 b$ & $21 \mathrm{a}$ & $21 b$ & $22 \mathrm{a}$ & $22 b$ \\
\hline 0.2 & 1.56 & 1.56 & 0.78 & 0.78 & 0.78 & 0.78 & 0.78 & 0.78 & 0.78 & 0.39 \\
\hline 50 & $>100$ & $>100$ & $>100$ & $>100$ & $>100$ & $>100$ & $>100$ & $>100$ & $>100=$ & $>100$ \\
\hline$<0.2$ & 0.78 & 1.56 & 0.39 & 0.39 & 0.2 & 0.2 & $<0.2$ & $<0.2$ & $<0.2$ & $<0.2$ \\
\hline$>100$ & $>100$ & $>100$ & $>100$ & $>100$ & $>100$ & $>100$ & $>100$ & $>100$ & $>100=$ & $>100$ \\
\hline$>100$ & $>100$ & $>100$ & $>100$ & $>100$ & $>100$ & $>100$ & $>100$ & $>100$ & $>100$ & $>100$ \\
\hline 1.56 & 3.12 & 3.12 & 0.78 & 0.78 & $\quad 0.39$ & 0.39 & 0.78 & 0.78 & $3 \quad 1.56$ & 1.56 \\
\hline 0.39 & 1.56 & 1.56 & 0.78 & 0.78 & $\quad 0.39$ & 0.39 & 0.78 & 0.78 & 0.78 & 0.78 \\
\hline$>100$ & $>100$ & $>100$ & $>100$ & $>100$ & $>100$ & $>100$ & $>100$ & $>100$ & $>100=$ & $>100$ \\
\hline$<0.2$ & 0.2 & 0.2 & $<0.2$ & $<0.2$ & $<0.2$ & $<0.2$ & $<0.2$ & $<0.2$ & $<0.2$ & $<0.2$ \\
\hline$<0.2$ & 0.2 & 0.39 & $<0.2$ & 0.2 & $<0.2$ & 0.2 & 0.2 & 0.2 & 0.78 & 0.78 \\
\hline$<0.2$ & 0.2 & 3.12 & 0.2 & 0.78 & 0.39 & 0.78 & 0.39 & 0.39 & $<0.2$ & 0.2 \\
\hline 25 & 6.25 & 25 & 6.25 & 12.5 & 3.12 & 12.5 & 12.5 & 25 & 25 & 50 \\
\hline 25 & 25 & 100 & 25 & 100 & 25 & 100 & 50 & $>100$ & 50 & $>100$ \\
\hline 25 & 25 & 100 & 25 & 100 & 50 & 100 & 25 & 100 & 25 & 100 \\
\hline 25 & 25 & 100 & 50 & $>100$ & 25 & $>100$ & 50 & 100 & 50 & 100 \\
\hline 25 & 25 & $>100$ & 50 & 100 & 25 & 100 & 50 & $>100$ & 50 & $>100$ \\
\hline 25 & 25 & $>100$ & 50 & 100 & 25 & 100 & 50 & 100 & 50 & 100 \\
\hline 6.25 & 6.25 & 50 & 25 & 50 & 12.5 & 50 & 12.5 & 100 & 50 & 100 \\
\hline 6.25 & 1.56 & 3.12 & 1.56 & 1.56 & 1.56 & 0.78 & 1.56 & 3.12 & 1.56 & 3.12 \\
\hline 0.78 & 0.39 & 0.78 & 0.39 & 0.39 & 0.39 & 0.78 & 0.39 & 0.78 & 0.39 & 0.78 \\
\hline 1.56 & 0.78 & 1.56 & 0.78 & 0.39 & 0.78 & 0.78 & 1.56 & $6 \quad 1.56$ & 1.56 & 3.12 \\
\hline 50 & 25 & $>100$ & 25 & $>100$ & 25 & $>100$ & 100 & $>100$ & 100 & $>100$ \\
\hline$>100$ & $>100$ & $>100$ & 100 & $>100$ & 100 & $>100$ & $>100$ & $>100$ & $>100$ & $>100$ \\
\hline$>100$ & $>100$ & $>100$ & $>100$ & $>100$ & $>100$ & $>100$ & $>100$ & $>100$ & $>100$ & $>100$ \\
\hline 50 & 12.5 & $>100$ & 12.5 & $>100$ & 25 & $>100$ & 100 & $>100$ & 100 & $>100$ \\
\hline 6.25 & 6.25 & 50 & 12.5 & 50 & 12.5 & 50 & 25 & 50 & 25 & 50 \\
\hline 100 & 100 & 100 & $>100$ & 100 & $>100$ & 100 & 100 & 100 & 100 & 100 \\
\hline 8.06 & 9.17 & 23.1 & 8.94 & 15.3 & 7.86 & 14.6 & 11.0 & 17.0 & 12.2 & 18.3 \\
\hline
\end{tabular}

MIC of $\mathbf{1} \mathbf{b}^{1)}$ was 34.8 , and that of $\mathbf{1 3 b}$ was 15.3 .

furanyl- (21b) and 23-O-tetrahydropyranylmycaminosyl tylonolides (22b) were also prepared from mycaminosyl tylonolide (1) by a similar synthetic pathway.

The antibacterial spectra of the compounds prepared are shown in Table 1. The results show that the C-23-modifications described here generally gave rise to compounds more active than the parent compounds ( $\mathbf{1} \mathbf{a}$ and $\mathbf{1 b}$ ) in terms of their antibacterial activity, and it is clearly shown that the $4^{\prime}$-deoxy compounds are always superior to the corresponding 4'-hydroxyl compounds. Among those prepared, prominent compounds in terms of the antibacterial activity are 10a, 11a, 13a, and 19a, as shown by mean MIC ${ }^{5)}$ in Table 1 .
AKIHIRO TANAKA TSUTOMU TSUCHIYA Sumio Umezawa

Institute of Bioorganic Chemistry, 1614 Ida, Nakahara-ku, Kawasaki 211, Japan

\section{Masa Hamada Hamao Umezawa}

Institute of Microbial Chemistry, Kamiosaki, Shinagawa-ku, Tokyo 141, Japan

(Received June 25, 1981) 


\section{References}

1) Tanaka, A.; T. Tsuchiya, S. Umezawa \& $\mathrm{H}$. Umezawa: Synthesis of 4'-deoxymycaminosyl tylonolide. J. Antibiotics 34: 1374 1376, 1981

2) Takana, A.; A. Watanabe, R. Kobayashi, T. Tsuchiya \& S. Umezawa: Syntheses of recyclized macrolide antibiotics and related derivatives from mycaminosyl tylonolide. Bull. Chem. Soc. Jpn. in press

3) Anisuzzaman, A. K. M. \& R. L. Whistler: Selective replacement of primary hydroxyl groups in carbohydrates; preparation of some carbo- hydrate derivatives containing halomethyl groups. Carbohydr. Res. 61: 511 518, 1978

4) Miyazaki, M.; A. Yoshikoshi \& P. A. Grieco: Pyridinium $p$-toluenesulfonate. A mild and efficient catalyst for the tetrahydropyranylation of alcohols. J. Org. Chem. 42: 3772 3774, 1977

5) Tsuchiya, T.; T. Jikihara, T. Miyake, S. Umezawa, M. Hamada \& H. Umezawa: 3'Deoxyamikacin and $3^{\prime}, 4^{\prime}$-dideoxyamikacin and their antibacterial activities. J. Antibiotics 32: $1351 \sim 1353,1979$ 